\title{
CONTACT STRUCTURES WITH DISTINCT HEEGAARD FLOER INVARIANTS
}

\author{
Olga Plamenevskaya
}

\begin{abstract}
We use the Heegaard Floer theory developed by P. Ozsváth and Z. Szabó to give a new proof of a theorem of P. Lisca and G. Matić. In particular, we prove that the contact structures on $Y=\partial X$ induced by non-homotopic Stein structures on the 4-manifold $X$ have distinct Heegaard Floer invariants. Our examples also show that Heegaard Floer homology can distinguish between non-isotopic tight contact structures.
\end{abstract}

\section{Introduction}

In $[\mathrm{LM}]$, Lisca and Matić gave examples of non-isotopic contact structures which are homotopic as plane fields. Using Seiberg-Witten theory, they proved

Theorem 1. [LM] Let $W$ be a smooth compact 4-manifold with boundary, equipped with two Stein structures $J_{1}, J_{2}$ with associated $\operatorname{Spin}^{c}$ structures $\mathfrak{s}_{1}, \mathfrak{s}_{2}$. If the induced contact structures on $\partial W$ are isotopic, then the $\operatorname{Spin}^{c}$ structures $\mathfrak{s}_{1}$ and $\mathfrak{s}_{2}$ are isomorphic.

In this paper we study Heegaard Floer contact invariants of such contact structures. These contact invariants were introduced by Ozsváth and Szabó in [OS5]; to an oriented contact 3 -manifold $(Y, \xi)$ with a co-oriented contact structure $\xi$ they associate an element $c(\xi)$ of the Heegaard Floer homology group $\widehat{H F}(-Y)$. Conjecturally, Heegaard Floer homology is equivalent to SeibergWitten Floer homology, and the Heegaard Floer contact invariants are the same as the Seiberg-Witten invariants of contact structures constructed in $[\mathrm{KM}]$. In the Heegaard Floer context, we can make the theorem of Lisca and Matić more precise:

Theorem 2. Let $W$ be a smooth 4-manifold with boundary, equipped with two Stein structures $J_{1}, J_{2}$ with associated $\operatorname{Spin}^{c}$ structures $\mathfrak{s}_{1}, \mathfrak{s}_{2}$, and let $\xi_{1}, \xi_{2}$ be the induced contact structures on $Y=\partial W$. If the $\operatorname{Spin}^{c}$ structures $\mathfrak{s}_{1}$ and $\mathfrak{s}_{2}$ are not isomorphic, then the contact invariants $c\left(\xi_{1}\right), c\left(\xi_{2}\right)$ are distinct elements of $\widehat{H F}(-Y)$.

There is some additional structure on Heegaard Floer homology groups:

$\widehat{H F}(Y)$ decomposes as a direct sum $\bigoplus_{\mathfrak{s}} \widehat{H F}(Y, \mathfrak{s})$ with summands corresponding to $\operatorname{Spin}^{c}$ structures on $Y$; if $c_{1}(\mathfrak{s})$ is torsion, the group $\widehat{H F}(Y, \mathfrak{s})$ is graded.

Received March 4, 2004. 
If the contact structures $\xi_{1}$ and $\xi_{2}$ are homotopic as plane fields, they induce the same $\operatorname{Spin}^{c}$ structure $\mathfrak{s}$, and the contact invariants $c\left(\xi_{1}\right), c\left(\xi_{2}\right)$ both lie in $\widehat{H F}(-Y, \mathfrak{s})$. In the torsion case, they also have the same grading. However, $c\left(\xi_{1}\right)$ and $c\left(\xi_{2}\right)$ can be nevertheless different, as follows from Theorem 2.

\section{Preliminaries on Heegaard Floer Homology}

In this section we briefly recall some necessary facts from the papers of Ozsváth and Szabó [OS1] - [OS6].

Given an oriented 3-manifold $Y$ equipped with a $\operatorname{Spin}^{c}$ structure $\mathfrak{t}$, there are homology groups $H F^{+}(Y, \mathfrak{t}), H F^{-}(Y, \mathfrak{t}), \widehat{H F}(Y, \mathfrak{t})$. The last one is the simplest, but we will mostly need the first two in this paper; the reader is referred to [OS1], [OS2] for the definitions and properties. A cobordism between two 3-manifolds induces a map on homology. More precisely, if $W$ is a cobordism from $Y_{1}$ to $Y_{2}$, and $\mathfrak{s}$ is a $\operatorname{Spin}^{c}$ structure on $W$ with restrictions $\mathfrak{s}\left|Y_{1}, \mathfrak{s}\right| Y_{2}$ on $Y_{1}, Y_{2}$, then there are maps $F_{W, \mathfrak{s}}^{\circ}: H F^{\circ}\left(Y_{1}, \mathfrak{s} \mid Y_{1}\right) \rightarrow H F^{\circ}\left(Y_{2}, \mathfrak{s} \mid Y_{2}\right)\left(H F^{\circ}\right.$ stands for one of the $\left.H F^{+}, H F^{-}, \widehat{H F}\right)$. These maps satisfy the composition law:

Proposition 1. [OS3] Let $W_{1}$ be a cobordism from $Y_{1}$ to $Y_{2}, W_{2}$ a cobordism from $Y_{2}$ to $Y_{3}$, and $W=W_{1} \cup_{Y_{2}} W_{2}$ the composite cobordism. Let $\mathfrak{s}_{i} \in \operatorname{Spin}^{c}\left(W_{i}\right)$, $i=1,2$ be two Spin $^{c}$ structures with $\mathfrak{s}_{1}\left|Y_{1}=\mathfrak{s}_{2}\right| Y_{2}$. Then for some choice of signs

$$
F_{W_{2}, \mathfrak{s}_{2}}^{\circ} \circ F_{W_{1}, \mathfrak{s}_{1}}^{\circ}=\sum_{\left\{\mathfrak{s} \in \operatorname{Spin}^{c}(W): \mathfrak{s}\left|W_{1}=\mathfrak{s}_{1}, \mathfrak{s}\right| W_{2}=\mathfrak{s}_{2}\right\}} \pm F_{W, \mathfrak{s}}^{\circ} .
$$

For a cobordism $W$ from $Y_{1}$ to $Y_{2}$ with $b_{2}^{+}(W)>1$, there is also a mixed invariant $F_{W, \mathfrak{s}}^{\operatorname{mix}}: H F^{-}\left(Y_{1}, \mathfrak{s} \mid Y_{1}\right) \rightarrow H F^{+}\left(Y_{2}, \mathfrak{s} \mid Y_{2}\right)[\mathrm{OS} 3]$. It is defined by taking an "admissible cut" $N$, which separates $W$ into cobordisms $X_{1}$ from $Y_{1}$ to $N$ and $X_{2}$ from $N$ to $Y_{2}$ with $b_{2}^{+}\left(X_{i}\right)>0$, and composing $F_{X_{1}}^{-}: H F^{-}\left(Y_{1}\right) \rightarrow H F^{-}(N)$ and $F_{X_{2}}^{+}: H F^{+}(N) \rightarrow H F^{+}\left(Y_{2}\right)$ in a certain way. We skip the details, as we can simply fix some admissible cut in our constructions. A "mixed" version of the composition law says that for cobordisms $W_{1}$ and $W_{2}$ as in Proposition 1

$$
F_{W_{2}, \mathfrak{s}_{2}}^{+} \circ F_{W_{1}, \mathfrak{s}_{1}}^{\operatorname{mix}}=\sum_{\left\{\mathfrak{s} \in \operatorname{Spin}^{c}(W): \mathfrak{s}\left|W_{1}=\mathfrak{s}_{1}, \mathfrak{s}\right| W_{2}=\mathfrak{s}_{2}\right\}} \pm F_{W, \mathfrak{s}}^{\text {mix }}
$$

whenever $b_{2}^{+}\left(W_{1}\right)>1$.

If $\mathfrak{s}$ is a torsion $\operatorname{Spin}^{c}$ structure, the homology groups $H F^{\circ}(Y, \mathfrak{s})$ are graded; the grading takes values in $\mathbb{Q}$ and changes under cobordisms according to the following dimension formula.

Proposition 2. [OS3] If $W$ is a cobordism from $Y_{1}$ to $Y_{2}$ endowed with a $\operatorname{Spin}^{c}$ structure whose restriction to $Y_{1}$ and $Y_{2}$ is torsion, then

$$
\tilde{\operatorname{gr}}\left(F_{W, \mathfrak{s}}^{\circ}(\xi)\right)-\tilde{\operatorname{gr}}(\xi)=\frac{c_{1}(\mathfrak{s})^{2}-2 \chi(W)-3 \sigma(W)}{4}
$$


for any homogeneous element $\xi$.

It follows that $F_{W, \mathfrak{s}}^{\operatorname{mix}}$ affects the gradings in the same way.

A closed 4-manifold $X$ can be punctured in two points and regarded as a cobordism from $S^{3}$ to $S^{3}$; if $b_{2}^{+}(X)>1$, the mixed invariant of this cobordism gives a closed manifold invariant $\Phi(X)$. If $X$ is symplectic, this invariant satisfies an important non-vanishing theorem [OS6]. Below we state a version of this theorem for Lefschetz fibrations, rephrasing it in terms of mixed invariants for convenience. Abusing notation, we denote by $X$ both the closed manifold and the corresponding cobordism from sphere to sphere. Recall that $H F^{ \pm}\left(S^{3}\right)$ are given by

$$
H F_{k}^{-}\left(S^{3}\right)=\mathbb{Z} \text { in gradings } k<0, k \text { even; } H F_{k}^{+}\left(S^{3}\right)=\mathbb{Z}, k \geq 0, k \text { even. }
$$

Theorem 3. [OS6] Let $\pi: X \rightarrow S^{2}$ be a relatively minimal Lefschetz fibration over the sphere with generic fiber $F$ of genus $g>1$, and $b_{2}^{+}(X)>1$. Then for the canonical $\operatorname{Spin}^{c}$ structure $k$ the map $F_{X, k}^{\operatorname{mix}}$ sends the generator of $\mathrm{HF}_{-2}^{-}\left(S^{3}\right)$ to the generator of $\mathrm{HF}_{0}^{+}\left(S^{3}\right)$ (and vanishes on the rest of $\mathrm{HF}^{-}\left(S^{3}\right)$ ).

For any other $\operatorname{Spin}^{c}$ structure $\mathfrak{s} \neq k$ with $\left\langle c_{1}(\mathfrak{s}),[F]\right\rangle \leq 2-2 g=\left\langle c_{1}(k),[F]\right\rangle$ the map $F_{X, \mathfrak{s}}^{\operatorname{mix}}$ vanishes.

Finally, we need to recall the construction of the invariant $c(\xi)$ for a contact manifold $(Y, \xi)$. We only consider co-oriented contact structures on $Y$. In [OS5], $c(\xi)$ is defined as an element of $\widehat{H F}(-Y)$; we will need to alter the definition slightly and look at $c(\xi) \in H F^{+}(-Y)$. The two elements obviously correspond to each other under the natural map $\widehat{H F}(-Y) \rightarrow H F^{+}(-Y)$. The definition uses the open book decomposion of $(Y, \xi)$, as well as the following fact.

Proposition 3. [OS6] Let $Y_{0}$ be a fibration over the circle whose fiber $F$ has genus $g>1$. Let $\mathfrak{k}$ be the canonical $\mathrm{Spin}^{c}$ structure induced by the tangent planes to the fibers. Then

$$
H F^{+}\left(Y_{0}, \mathfrak{k}\right)=\mathbb{Z},
$$

and $H F^{+}\left(Y_{0}, \mathfrak{s}\right)=0$ for any other $\mathfrak{s}$ with $\left\langle c_{1}(\mathfrak{s}),[F]\right\rangle=2-2 g=\left\langle c_{1}(\mathfrak{k}),[F]\right\rangle$.

As proven by Giroux [Gi1], contact manifolds can be described in terms of open books. An open book decomposion of $Y$ is a pair $(K, p)$ consisting of a (fibered) knot $K \subset Y$ and a fibration $p: Y \backslash K \rightarrow S^{1}$ whose fibers $p^{-1}(\phi)$ are interiors of compact embedded surfaces $F_{\phi}$ bounded by $K . K$ is then called the binding of the open book, and the fibers are the pages. An open book is compatible with a contact structure $\xi$ given by a contact form $\alpha$ on $Y$, if $\mathrm{d} \alpha$ is an area form on each page, and the binding is transverse to the contact planes and oriented as the boudary of $(F, \mathrm{~d} \alpha)$. There is a one-to-one correspondence between isotopy classes of contact structures and the open books up to stabilization [Gi1].

Given a compatible open book for $(Y, \xi)$, we can obtain a fibration $Y_{0}$ by performing 0 -surgery on the binding. Let $V_{0}$ the corresponding cobordism from $Y$ to $Y_{0}$, which can also be regarded as a cobordism from $-Y_{0}$ to $-Y$. The 
canonical $\operatorname{Spin}^{c}$ structure $\mathfrak{k}$ on $Y_{0}$ determines a $\operatorname{Spin}^{c}$ structure for $V_{0}$, so we can drop it from notation below.

Definition 1. [OS5, Proposition 3.1] The contact invariant is defined as

$$
c(\xi)=F_{V_{0}}^{+}(c),
$$

where $c$ stands for a generator of $\mathrm{HF}^{+}\left(Y_{0}, \mathfrak{k}\right)$, and $c(\xi)$ is defined up to sign.

It is proven in [OS5] that $c(\xi)$ is independent of the choice of the open book.

2.1. Contact Invariants and Concave Fillings. In this section we study contact invariants by using concave fillings of contact manifolds, and prove Theorem 2. More precisely, we prove

Theorem 4. Let $W$ be a smooth compact 4-manifold with boundary $Y=\partial X$. Let $J_{1}, J_{2}$ be two Stein structures on $W$ that induce $\operatorname{Spin}^{c}$ structures $\mathfrak{s}_{1}, \mathfrak{s}_{2}$ on $W$ and contact structures $\xi_{1}, \xi_{2}$ on $Y$. We puncture $W$ and regard it as a cobordism from $-Y$ to $S^{3}$. Suppose that $\mathfrak{s}_{1}\left|Y=\mathfrak{s}_{2}\right| Y$, but the $\operatorname{Spin}^{c}$ structures $\mathfrak{s}_{1}, \mathfrak{s}_{2}$ are not isomorphic. Then

1. $F_{W, \mathfrak{s}_{i}}^{+}\left(c\left(\xi_{j}\right)\right)=0$ for $i \neq j$;

2. $F_{W, \mathfrak{s}_{i}}^{+}\left(c\left(\xi_{i}\right)\right)$ is a generator of $H F_{0}^{+}\left(S^{3}\right)$.

Obviously, Theorem 4 implies Theorem 2 after we switch between the invariants in $\widehat{H F}(-Y)$ and their images in $H F^{+}(-Y):$ if $\mathfrak{s}_{1}\left|Y \neq \mathfrak{s}_{2}\right| Y$, then the contact elements $c\left(\xi_{1}\right)$ and $c\left(\xi_{2}\right)$ lie in the components of $H F^{+}(-Y)$ corresponding to different $\operatorname{Spin}^{c}$-structures, and the statement of Theorem 2 is trivial.

Corollary 1. Suppose that a 3-manifold $Y$ bounds a compact smooth 4-manifold $X$. If $X$ supports $n$ pairwise non-homotopic Stein structures, then

$$
\operatorname{rk}(\widehat{H F}(Y)) \geq n \text {. }
$$

This follows from Theorem 4 and the duality $\widehat{H F}^{*}(-Y) \cong \widehat{H F}_{*}(Y)$, which gives an isomorphism $\widehat{H F}(-Y) \cong \widehat{H F}(Y)$ in the non-torsion case [OS1].

For a Stein fillable contact manifold $(Y, \xi)$, we want to describe $c(\xi)$ as a mixed invariant of a certain concave filling of $(Y, \xi)$. We construct this concave filling, following the work of Akbulut and Ozbagci [AO2].

Suppose that $W$ is a (convex) Stein filling of $(Y, \xi)$. First we need to represent $W$ as a positive allowable Lefschetz fibration [AO1], whose generic fiber is a surface with boundary. This induces an open book decomposition of $(Y, \xi)$ with monodromy consisting of non-separating positive Dehn twists. The original Stein structure on $W$ can be recovered from the Lefschetz fibration, and the open book is compatible with $\xi$. Note that the compatibility does not directly follow from the argument in [AO1]; for completeness we review this construction in Appendix, strengthening it slightly and proving the compatibility statement.

Given a structure of positive allowable Lefschetz fibration on $W$ and the induced open book decomposition of $Y$, we perform 0-surgery on the binding to get a cobordism $V_{0}$ from $Y$ to $Y_{0}$. Now $W \cup V_{0}$ is a Lefschetz fibration over the 
disk, whose regular fiber $F$ is a closed surface obtained by capping off the page of the open book, and $Y_{0}$ is a fibration over the circle. The monodromy of $Y_{0}$ comes from the open book, and can be represented as a product $c_{1} c_{2} \ldots c_{k}$ of positive Dehn twists. We need a concave filling for $Y_{0}$, so we want to construct a Lefschetz fibration with monodromy $c_{k}^{-1} \ldots c_{1}^{-1}$. Recalling that the mapping class group of a closed surface is generated by non-separating positive Dehn twists [AO2], we rewrite $c_{k}^{-1} \ldots c_{1}^{-1}$ as a product of such twists. Putting in a node of the Lefschetz fibration for each positive Dehn twist in the monodromy gives a Lefschetz fibration $V_{1}$ with $\partial V_{1}=-Y_{0}$.

To use the mixed invariants, we must have $b_{2}^{+}\left(V_{1}\right)>1$. This can be achieved by a trick from [AO2]: consider a Lefschetz fibration $G$ over the disk with the nodes defined by Dehn twists of the word $\left(a_{1} b_{1} a_{2} b_{2} \ldots a_{g} b_{g}\right)^{4 g+2}$; here $a_{i}, b_{i}$ stand for positive Dehn twists around the curves shown in Figure 1. Since this

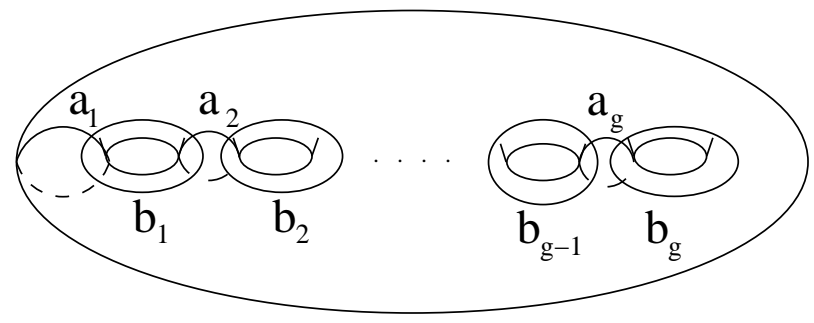

Figure 1. $\left(a_{1} b_{1} a_{2} b_{2} \ldots a_{g} b_{g}\right)^{4 g+2}=1$

word is equivalent to identity [Bi], we can glue a copy of $G$ into our fibration $V_{1}$ without affecting the monodromy of the boundary $\partial V_{1}=-Y_{0}$. On the other hand, a Lefschetz fibration has a symplectic structure [GS], so $b_{2}^{+}(G)>0$. Gluing in two copies of $G$, we may assume that $b_{2}^{+}\left(V_{1}\right)>1$.

By construction, $X=W \cup V_{0} \cup V_{1}$ is a Lefschetz fibration over the sphere, and $V=V_{0} \cup V_{1}$ is a concave symplectic filling of $(Y, \xi)$. Let $k$ denote the canonical $\operatorname{Spin}^{c}$ structure on $X$; we also write $k$ for its restrictions to $V, V_{1}$ etc.

Regard $V$ as a cobordism from $S^{3}$ to $-Y$, puncturing it at a point. The following fact is implicitly mentioned in [OS5].

Lemma 1. Suppose $c_{1}(\xi)$ is torsion. Let $\theta$ be the generator of $\mathrm{HF}_{-2}^{-}\left(S^{3}\right)$. Then

$$
c(\xi)= \pm F_{V, k}^{\operatorname{mix}}(\theta) .
$$

Proof. As before, let $c$ be the generator of $\mathrm{HF}^{+}\left(-Y_{0}\right)$. Observe that $F_{V_{1}}^{\operatorname{mix}}(\theta)=$ $\pm c$. Indeed, by (2) we have

$$
F_{V_{0} \cup W, k}^{+} \circ F_{V_{1}, k}^{\text {mix }}=\sum_{\left\{\mathfrak{s} \in \operatorname{Spin}^{c}(X):\right.} \sum_{\left.\mathfrak{s}\left|V_{0} \cup W=k, \mathfrak{s}\right| V_{1}=k\right\}} \pm F_{X, s}^{\mathrm{mix}}= \pm F_{X, k}^{\operatorname{mix}}
$$

(there is just one term that survives in the sum, because $X$ is a symplectic fibration, and the non-canonical $\operatorname{Spin}^{c}$ structures with $\left\langle c_{1}(\mathfrak{s}),[F]\right\rangle=2-2 g$ give 
zero maps by Theorem 3). So $F_{V_{0} \cup W}^{+} \circ F_{V_{1}}^{\operatorname{mix}}(\theta)$ is the generator of $H F_{0}^{+}\left(S^{3}\right)$, but this map factors through $H F^{+}\left(-Y_{0}, \mathfrak{k}\right)=\mathbb{Z}$, so we must have $F_{V_{1}}^{\operatorname{mix}}(\theta)= \pm c$.

Now it follows that

$$
c(\xi)= \pm F_{V_{0}}^{+} \circ F_{V_{1}, k}^{\mathrm{mix}}(\theta)=\sum_{\left\{\mathfrak{s} \in \operatorname{Spin}^{c}(V): \mathfrak{s}\left|V_{0}=k, \mathfrak{s}\right| V_{1}=k\right\}} \pm F_{V, \mathfrak{s}}^{\mathrm{mix}}(\theta) .
$$

Because $V_{0}$ consists of one 2-handle attachment, the $\operatorname{Spin}^{c}$-structures with given restrictions to $V_{0}$ and $V_{1}$ are of the form $k+n \mathrm{PD}[F], n \in \mathbb{Z}$. The dimension formula (3) now implies that all non-zero terms in the sum (4) must have different absolute gradings, since $c_{1}(k+n \mathrm{PD}[F])^{2}=c_{1}(k)^{2}+2 n(2-2 g)$, and $g>1$. However, it is clear from the definition that the contact invariant $c(\xi)$ is a homogeneous element in homology, so only one summand can be non-trivial. This summand has to be $F_{V, k}^{\operatorname{mix}}(\theta)$ : again we can use the composition law (2) and Theorem 3 to write

$$
F_{W, k}^{+} \circ F_{V, k}^{\operatorname{mix}}=\sum_{\left\{\mathfrak{s} \in \operatorname{Spin}^{c}(X): \mathfrak{s}\left|V_{0} \cup W=k, \mathfrak{s}\right| V_{1}=k\right\}} \pm F_{X, \mathfrak{s}}^{\mathrm{mix}}= \pm F_{X, k}^{\operatorname{mix}},
$$

so $F_{W, k}^{+} \circ F_{V, k}^{\operatorname{mix}}(\theta)$ is the generator of $H F_{0}^{+}\left(S^{3}\right)$, and it follows that $F_{V, k}^{\operatorname{mix}}(\theta) \neq 0$. Then $F_{V, k}^{\operatorname{mix}}(\theta)= \pm c(\xi)$.

Proof of Theorem 4. We first deal with the case where $c_{1}\left(\mathfrak{s}_{i} \mid Y\right)$ is torsion. Consider the Lefschetz fibration decomposition of the Stein manifold $\left(W, J_{1}\right)$, and construct the concave filling $V$ as above for the contact structure $\xi=\xi_{1}$. As before, the two pieces $V$ and $W$ fit together to form a Lefschetz fibration $X$ over the sphere; by construction, $\mathfrak{s}_{1}=k$ on $W$. Looking at the proof of Lemma 1, we can use (5) to show that $F_{W, \mathfrak{s}_{1}}^{+} \circ F_{V, k}^{\text {mix }}$ is the generator of $H F_{0}^{+}\left(S^{3}\right)$; by Lemma 1 itself, $F_{V, k}^{\operatorname{mix}}(\theta)= \pm c\left(\xi_{1}\right)$, and Part (2) of the Theorem follows.

To prove Part (1), endow $W$ with the $\operatorname{Spin}^{c}$ structure $\mathfrak{s}_{2}$, and glue it to the concave filling $V$ of the contact structure $\xi=\xi_{1}$. Of course, we get the manifold $X$, which topologically remains the same, and the $\operatorname{Spin}^{c}$ structures can be put together as $\mathfrak{s}_{1}\left|Y=\mathfrak{s}_{2}\right| Y$, but we no longer get the canonical $\operatorname{Spin}^{c}$ structure associated to the symplectic structure. Again by Lemma 1 and the composition law,

$$
F_{W, \mathfrak{s}_{2}}^{+}\left(c\left(\xi_{1}\right)\right)= \pm F_{W, \mathfrak{s}_{2}}^{+} \circ F_{V, k}^{\operatorname{mix}}(\theta)=\sum_{\left\{\mathfrak{s} \in \operatorname{Spin}^{c}(X): \mathfrak{s}\left|W=\mathfrak{s}_{2}, \mathfrak{s}\right| V=k\right\}} \pm F_{X, \mathfrak{s}}^{\operatorname{mix}}(\theta) .
$$

For each of the $\operatorname{Spin}^{c}$ structures in the sum we still have $\left\langle c_{1}(\mathfrak{s}),[F]\right\rangle=2-2 g$, but now none of them is canonical, since $\mathfrak{s}_{2}$ is different from $\mathfrak{s}_{1}$. By Theorem 3 , every term in the sum is zero.

We have proved Theorem 4 for the torsion case; it remains to treat the case when $c_{1}\left(\xi_{i}\right)$ is non-torsion. The dimension formula is no longer valid and we 
can't use Lemma 1, but we can look at the same construction and write

$$
F_{W, \mathfrak{s}_{i}}^{+}\left(c\left(\xi_{1}\right)\right)= \pm F_{W, \mathfrak{s}_{i}}^{+} \circ F_{V_{0}, k}^{+} \circ F_{V_{1}, k}^{\operatorname{mix}}(\theta)=\sum_{\mathfrak{s}} \pm F_{X, \mathfrak{s}}^{\operatorname{mix}}(\theta),
$$

where the sum is now taken over all $\operatorname{Spin}^{c}$ structures on $X$ which restrict to $W$ as $\mathfrak{s}_{i}$ and to $V_{0}$ and $V_{1}$ as $k$. As before, all these $\operatorname{Spin}^{c}$ structures have $\left\langle c_{1}(\mathfrak{s}),[F]\right\rangle=$ $2-2 g$. If $\mathfrak{s}_{i}=\mathfrak{s}_{1}$, one of the terms in (6) corresponds to the canonical $\operatorname{Spin}^{c}$ structure on $X$, and the sum is equal to the generator of $H F_{0}^{+}\left(S^{3}\right)$; if $\mathfrak{s}_{i}=\mathfrak{s}_{2}$, all the resulting $\operatorname{Spin}^{c}$ structures on $X$ are different from the canonical $\operatorname{Spin}^{c}$ structure, so the sum is zero.

\section{An Example}

We now look at an example due to Lisca and Matić [LM].

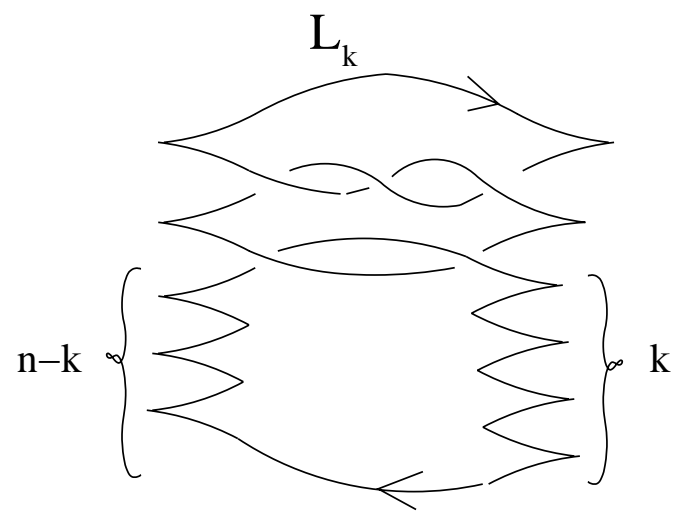

FIGURE 2. Legendrian link $L_{k}$

Example. Let the contact manifold $\left(Y_{n}, \xi_{k}\right)$ be obtained as a Legendrian surgery on the (oriented) Legendrian link $L_{k}$ shown on Fig. $2(k$ kinks on the right and $n-k$ kinks on the left give $r=2 k-n$ for the rotation number of the unknot; the rotation number of the trefoil is 0 ). Varying $k$, we get $n-1$ contact structures $\xi_{1}, \ldots, \xi_{n-1}$ on $Y_{n}$.

Topologically, the manifold $Y_{n}$ is the Brieskorn homology sphere $\Sigma(2,3,6 n-$ 1) with the orientation reversed; it is the boundary of the nucleus $N_{n}$. The Legendrian surgery cobordism corresponding to $L_{k}$ endows $N_{n}$ with a Stein structure $J_{k}$. We have $c_{1}\left(J_{k}\right)=(2 k-n) \mathrm{PD}[T]$, where $T$ is formed by a Seifert surface for the trefoil and the core of the handle attached to it [LM], so $J_{k}$ are pairwise non-homotopic. The contact structures $\xi_{i}$ are all homotopic by Gompf's criterion [Go], since $Y$ is a homology sphere, and the Hopf invariant, defined as $h(\xi)=c_{1}(J)^{2}-2 \chi(W)-3 \sigma(W)$ for an almost-complex 4-manifold $(W, J)$ with boundary $(Y, \xi)$, is equal to -6 for all $\xi_{i}$. 
The manifold $Y$ can be obtained as $1 / n$-surgery on the right-handed trefoil, and we can compute (cf. Section 8 of [OS4])

$$
\widehat{H F}(-Y)=\widehat{H F}(-\Sigma(2,3,6 n-1))=\mathbb{Z}_{(+2)}^{n} \oplus \mathbb{Z}_{(+1)}^{n-1},
$$

where the subscripts indicate grading.

By [OS5], the grading of the contact invariant is related to the Hopf invariant by $\tilde{\operatorname{gr}}(c(\xi))=-h(\xi) / 4-\frac{1}{2}$, so for all $\xi_{i}$ the grading $\tilde{\operatorname{gr}}\left(c\left(\xi_{i}\right)\right)=+1$. Theorem 2 implies that the contact elements $c\left(\xi_{i}\right)$ are pairwise distinct; moreover, it follows from Theorem 4 that each $c\left(\xi_{i}\right)$ is a primitive element of $\widehat{H F}(-Y)$, and that $c\left(\xi_{1}\right), \ldots c\left(\xi_{n-1}\right)$ span $\mathbb{Z}_{(+1)}^{n-1} \subset \widehat{H F}(-Y)$.

Remark. The fact that the contact structures in this example have distinct contact invariants was also discovered by Paolo Lisca and András Stipsicz [LS], who have a different proof.

\section{Appendix A. Lefschetz fibrations on Stein manifolds with boundary}

The decompositions of Stein manifolds as positive allowable Lefschetz fibrations were constructed by Akbulut and Ozbagci in [AO1]. We give an overview of their construction here, modifying it slightly to suit our purposes, and taking particular care to prove that the open book induced by the Lefschetz fibration is compatible with the contact structure on the boundary of the Stein manifold.

By [El], [Go], a Stein manifold $W$ with boundary $\partial W=Y$ can be represented as $D^{4} \cup$ (1-handles) $\cup$ (2-handles); more precisely, $W$ is obtained by attaching $n$ 1-handles to the ball $D^{4}$ and extending the Stein structure on $D^{4}$ to the handles to get the (unique) Stein structure on $\#_{n} S^{1} \times D^{3}$; the 2 -handles are attached to components $L_{i}$ of a Legendrian link $L$ in $\#_{n} S^{1} \times D^{3}$, with the framings given by $\operatorname{tb}\left(L_{i}\right)-1\left(\operatorname{tb}\left(L_{i}\right)\right.$ denotes the Thurston-Bennequin number of $\left.L_{i}\right)$. In other words, $Y$ can be obtained by a Legendrian surgery on $L \subset \#_{n} S^{1} \times S^{2}$, so that the corresponding surgery cobordism is $W$.

To construct a Lefschetz fibration, we start with the case where $W$ has no 1-handles, so $Y$ is obtained as a Legendrian surgery on a Legendrian link $L$ in $S^{3}$.

Let $\xi_{0}$ denote the standard contact structure on $S^{3}$. The key ingredient of the construction is the following fact.

Proposition 4. Given a Legendrian link $L \subset S^{3}$, there exists an open book decomposition of $S^{3}$, such that:

1. the induced contact structure $\xi$ is isotopic to $\xi_{0}$;

2. the link $L$ is contained in one of the pages, and does not separate it;

3. $L$ is Legendrian with respect to $\xi$;

4. there exists an isotopy which fixes $L$ and takes $\xi$ to $\xi_{0}$, so the Legendrian type of the link is the same with respect to $\xi$ and $\xi_{0}$;

5. the framing of $L$ given by the page of the open book is the same as the contact framing. 
Note that (5) trivially follows from (1)-(4).

In [AO1] the statements (1), (2), and (5) are proved by putting $L$ into a "square bridge position" and constructing an open book for $S^{3}$ which contains this link. The binding of this open book is a torus knot, so the monodromy produces the standard contact structure on $S^{3}$. Note, however, that when $L$ is moved to the square bridge position, its Legendrian type is lost, and the contact structure forgotten; Parts (3) and (4) of Proposition 4 are unclear from [AO1].

Proof. We start by constructing one page of the open book. Puncturing the sphere at one point, we may consider links in $\left(\mathbb{R}^{3}, \xi_{0}\right)$; we assume that the contact structure $\xi_{0}$ on $\mathbb{R}^{3}$ is given by the contact form $\alpha_{0}=\mathrm{d} z+x \mathrm{~d} y$. The next lemma is very similar to Theorem 2 from [AO1], but keeps the link Legendrian and remembers the contact structure.

Lemma 2. Given a Legendrian link $L$ in $\left(\mathbb{R}^{3}, \xi_{0}\right)$, there exists a surface $F$ containing $L$, such that $\mathrm{d} \alpha_{0}$ is an area form for $F, \partial F=K$ is a torus knot transverse to $\xi_{0}$, and $L$ does not separate $F$.

Proof. After an appropriate Legendrian isotopy, we assume that the front projection of $L$ consists of segments which are straight lines (except in the neighborhoods of junctions), and all the negatively sloped segments have slope -1 , while the positive slopes are all equal to +1 (see Figure 3 ). Let $l_{i}=\left\{x=1, z=y+b_{i}\right\}$, $i=1, \ldots, p$ and $m_{j}=\left\{x=-1, z=-y+d_{j}\right\}, j=1, \ldots, q$ be the lines in $\mathbb{R}^{3}$ containing these segments; adding some extra lines if necessary, we can take $p$ and $q$ relatively prime. Denote by $t_{i, j}$ the intersection point of the lines $\left\{z=y+b_{i}\right\}$ and $\left\{z=-y+d_{j}\right\}$ on the $y z$-plane.

We start the contruction of $F$ by looking at the narrow strips

$$
\begin{aligned}
& S_{i}^{+}=\left\{1-\epsilon \leq x \leq 1+\epsilon, z=y+b_{i}\right\}, i=1, \ldots, p, \\
& S_{j}^{-}=\left\{-1-\epsilon \leq x \leq-1+\epsilon, z=-y+d_{j}\right\}, j=1, \ldots, q,
\end{aligned}
$$

surrounding the straight segments of knots. Taking the strips long enough, so that each of the points $\left( \pm 1, t_{i, j}\right)$ is contained in one of the strips, we obtain a grid similar to the one shown on Figure 4. If $\epsilon>0$ is small enough, $\mathrm{d} \alpha$ gives an area form on each strip. We connect the points $\left(1, t_{i, j}\right)$ and $\left(-1, t_{i, j}\right)$ for all $i, j$ by a segment of a straight line, and construct a band around this segment. The band connects the strips $S_{i}^{+}$and $S_{j}^{-}$; it twists by $90^{\circ}$ along the way, following the contact planes (see Figure 5). By construction, we get a surface $F$ whose boundary is a $(p, q)$-torus knot; it is also clear that $F$ is close enough to the contact planes, which means that $\mathrm{d} \alpha_{0}$ induces an area form on $F$.

The line segments of the Legendrian link $L$ lie on $F$, but $L$ might not be contained in $F$ around the junctions. However, we can perturb $F$ slightly, and move $L$ by a Legendrian isotopy to put it on $F$; obviously, $L$ is non-separating.

It remains to ensure that the boundary of $F$ is transverse to the contact planes. Since $d \alpha_{0}$ induces an area form on $F$, this is easy to achieve by moving the torus knot $K$ on this surface to make it transverse to the characterictic foliation on $F$ 


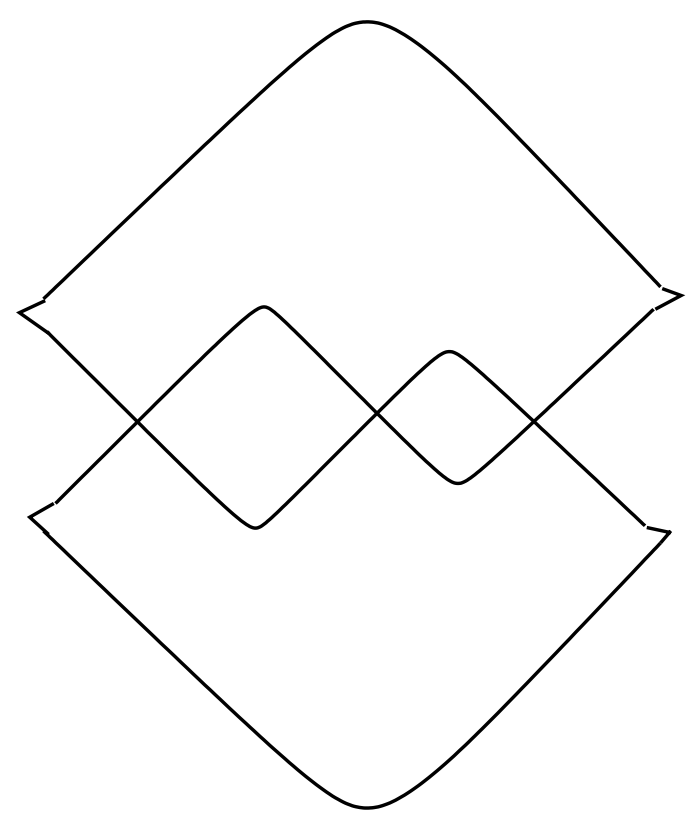

Figure 3. Legendrian link $L$

(we may assume that the singular points of this foliation are isolated, so they do not present a problem).

Now we can construct the required open book, starting with the page from Lemma 2. We immediately get an interval worth of pages, perturbing this page slightly and making sure that $\mathrm{d} \alpha_{0}$ is an area form on each newly constructed page. The pages will span a handlebody $H_{1}$ (a thickening of the original page).

Since the torus knot $K$ is fibered, we can fiber the complementary handlebody $H_{2}=S^{3} \backslash H_{1}$ by the pages with binding $K$, thus completing the picture to a fibration $\pi: S^{3} \backslash K \rightarrow S^{1}$. Unfortunately, the resulting open book does not have to be compatible with the contact form $\alpha_{0}$ : we have no guarantee that $d \alpha_{0}$ induces an area form on pages in $\mathrm{H}_{2}$.

However, we can find a contact form which is compatible with the open book $(K, \pi)$ and restricts to $\alpha_{0}$ on $H_{1}$ by using Thurston-Winkelnkemper construction [TW]. Denote by $\phi: F \rightarrow F$ the monodromy of the open book, assuming that $\phi=$ id in the neighborhood of the binding. Let $T_{\phi}$ be the mapping torus

$$
T_{\phi}=(F \times[0,1]) / \sim, \text { where }(x, 1) \sim(\phi(x), 0) .
$$

Cut out a small tubular neighborhood $K \times D^{2}$ of $K$ and shrink $F$ accordingly to represent the sphere as

$$
S^{3}=T_{\phi} \cup_{\partial} K \times D^{2}
$$




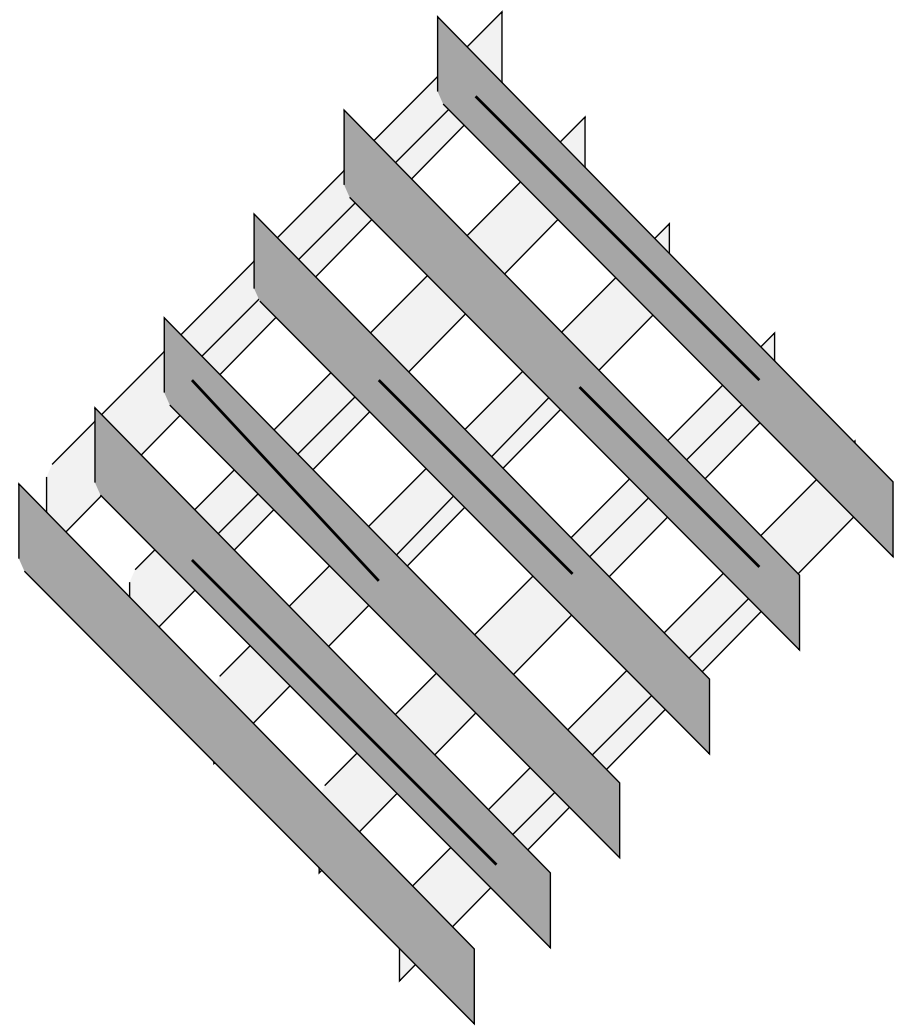

FIGURE 4. Strips of surface around L.

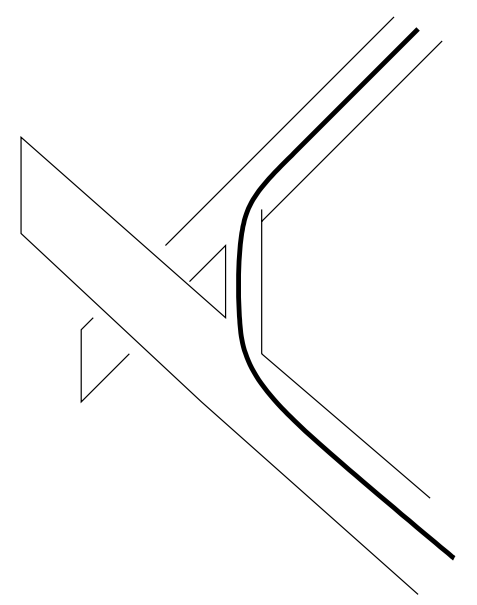

FiguRE 5. A connecting band.

We may assume that the handlebody $H_{1}$ consists of the pages $F \times\{t\}$ with $t \in\left[0, \frac{1}{2}\right] \subset S^{1}$, and that $\mathrm{d} \alpha_{0}$ gives an area form for all pages in the bigger handlebody $F \times\left[-\delta, \frac{1}{2}+\delta\right]$ for some small $\delta>0$. 
Let $\alpha^{t}$ be the restriction of the form $\alpha_{0}=\mathrm{d} z+x \mathrm{~d} y$ to the page $F \times\{t\}$. Set $\beta^{t}=2(1-t) \alpha^{\frac{1}{2}}+(2 t-1) \phi^{*} \alpha^{0}, t \in\left[\frac{1}{2}, 1\right]$; if $\kappa>0$ is large enough, $\beta^{t}+\kappa \mathrm{d} t$ is a contact form on $F \times\left[\frac{1}{2}, 1\right]$.

Let $\nu(t)$ be a positive increasing smooth function on $\left[\frac{1}{2}, \frac{1}{2}+\delta\right]$, such that $\nu\left(\frac{1}{2}\right)=0$, and $\nu\left(\frac{1}{2}+\delta\right)=\kappa$. The form $\alpha^{t}+\nu(t) \mathrm{d} t$ is contact on $F \times\left[\frac{1}{2}, \frac{1}{2}+\delta\right]$, and "connects" $\alpha_{0}$ and $\beta^{t}+\kappa \mathrm{d} t$. Construct $\alpha^{t}+\mu(t) \mathrm{d} t$ on $F \times[-\delta, 0]$ by analogy; now the forms

$$
\begin{array}{rll}
\alpha_{0} & \text { on } & F \times\left[0, \frac{1}{2}\right], \\
\alpha^{t}+\nu(t) \mathrm{d} t & \text { on } & F \times\left[\frac{1}{2}, \frac{1}{2}+\delta\right], \\
\beta^{t}+\kappa \mathrm{d} t & \text { on } & F \times\left[\frac{1}{2}+\delta, 1-\delta\right], \\
\alpha^{t}+\mu(t) \mathrm{d} t & \text { on } & F \times[-\delta, 0]
\end{array}
$$

fit together to produce a contact form on the mapping torus. This form extends over the binding, since the pages are transverse to the contact planes along the boundary.

As the binding of the open book $(K, \pi)$ is a torus knot, the monodromy is a product of non-separating positive Dehn twists [AO1], so the corresponding contact structure $\xi$ is Stein fillable. It follows that $\xi$ is isotopic to $\xi_{0}$. Moreover, the restrictions of $\xi$ and $\xi_{0}$ to the handlebody $H_{1}$ coincide, so the link $L$ obviously remains Legendrian for $\xi$, and Part (3) of the Proposition is established. To prove Part (4), we will show that on the handlebody $H_{2}$ the contact structures $\xi_{0}$ and $\xi$ are isotopic relative to the boundary (as they coincide on $H_{1}$, the restrictions of $\xi$ and $\xi_{0}$ to $\partial H_{2}=\partial H_{1}$ are the same). We will be using convex surfaces and dividing curves (see [Gi2], [Ho], [Ka]) in our proof. Note that we may perturb the surface $\partial H_{1}=\partial H_{2}$ slightly, and assume that it is convex.

Lemma 3. Assume that the handlebody $H \subset S^{3}$ is a thickening of the Seifert surface of a torus knot $K$. Consider tight contact structures on $H$ with convex boundary $\partial H$, for which the dividing set is $\Gamma=K$. Suppose that two tight contact structures $\xi, \zeta$ on $H$ induce the same characteristic foliation $\mathcal{F}$ on $\partial H$, and that $\mathcal{F}$ is adapted to $\Gamma$. Then $\xi$ and $\zeta$ are isotopic relative to $\partial H$.

Proof. A Seifert surface of the (p,q)-torus knot $K$ can be obtained by plumbing together $(p-1)(q-1)$ positive Hopf bands. The handlebody $H$ then decomposes as a boundary connected sum of thickened Hopf bands, which can be thought of as solid tori with convex boundary and dividing set $\Gamma$ given by two parallel curves with slope -1 . For any handlebody which is obtained by thickening of a plumbed sum of $n$ positive Hopf bands, we prove the statement of Lemma 3 by induction on $n$. The base of induction follows from Honda's classification of tight contact structures on the solid torus.

Lemma 4. [Ho] Let $\Gamma \subset T^{2}=\partial S^{1} \times D^{2}$ consist of two parallel curves with slope -1 . Then two tight contact structures on $S^{1} \times D^{2}$ with convex boundary 
$T^{2}$ are isotopic rel $T^{2}$ if they induce the same characterictic foliation adapted to $\Gamma$.

For the induction step, we want to cut one of the solid tori off the handlebody $H$. Choose an appropriate disk $\mathcal{D} \subset H$ with Legendrian boundary $\partial \mathcal{D} \subset \partial H$, so that $H=\left(S^{1} \times D^{2}\right) \cup_{\mathcal{D}} \tilde{H}$, where $\tilde{H}$ is a handlebody of smaller genus. To check that the dividing set is given by two curves of slope -1 on the boundary $S^{1} \times D^{2}$ and satisfies our assumption on the boundary of $\tilde{H}$, we examine the dividing curves on the cutting disk $\mathcal{D}$. Observe that $\partial \mathcal{D}$ meets $\Gamma$ in four points; we claim that after we cut along $\mathcal{D}$, round the corners of the resulting surfaces, and regard $\mathcal{D}$ as part of $\partial\left(S^{1} \times D^{2}\right)$ or $\partial H$, the dividing set inside $\mathcal{D}$ consists of two lines joining these points pairwise (for each surface). Indeed, otherwise $\Gamma$ would have a component bounding a disk inside $\mathcal{D}$, but this is a contradiction with Giroux's criterion:

Proposition 5. (Giroux) If $\Sigma \neq S^{2}$ is a convex surface (closed or compact with Legendrian boundary) in a contact manifold $(M, \xi)$, then $\Sigma$ has a tight neighborhood if and only if the dividing set $\Gamma_{\Sigma}$ has no homotopically trivial curves.
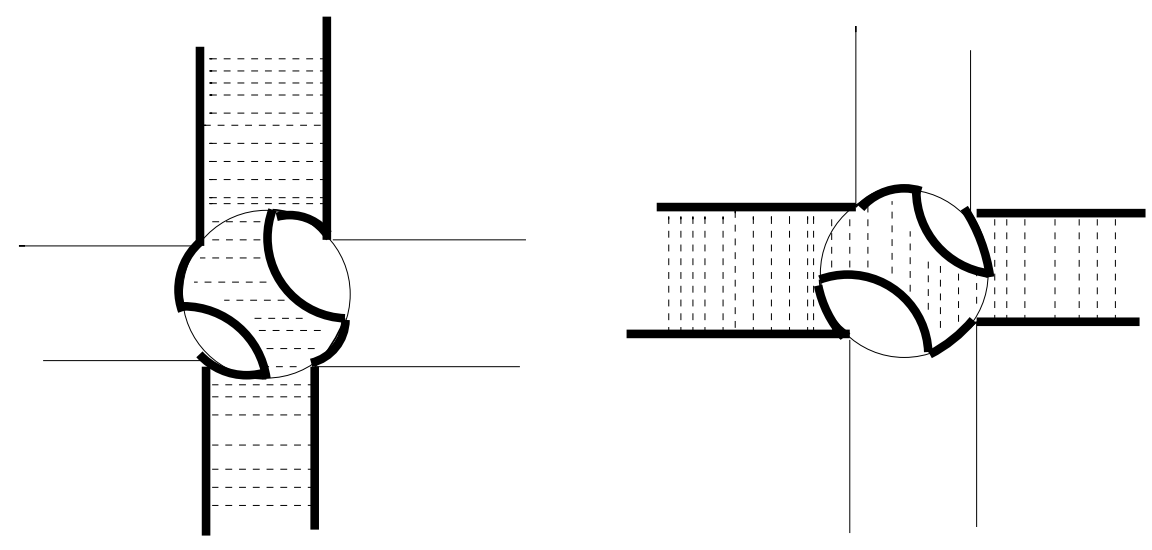

Figure 6. Correct dividing set.

There are two possible ways for the two lines to join four points; these are shown on Figures 6 and 7 (for the explanation of what happens to the dividing set when two convex surfaces meet and the corners are rounded, we refer the reader to Section 3.3.2 in [Ho]). It remains to observe that Figure 7 produces a homotopically trivial curve on the boundary of the solid torus and is ruled out by Giroux's criterion, while on Figure 6 the dividing set on $\mathcal{D}$ connects the bands as required, decomposing the "core surface" of $H$ into a plumbed sum of a Hopf band and a "core surface" for $\tilde{H}$. The uniqueness of the tight contact structures on $\tilde{H}$ and $S^{1} \times D^{2}$ (with given boundary conditions) now implies the uniqueness of the tight contact structure on $H$, and the induction step follows. (The dotted lines on Figures 6 and 7 are used to highlight the bands and do not encode any foliation). 

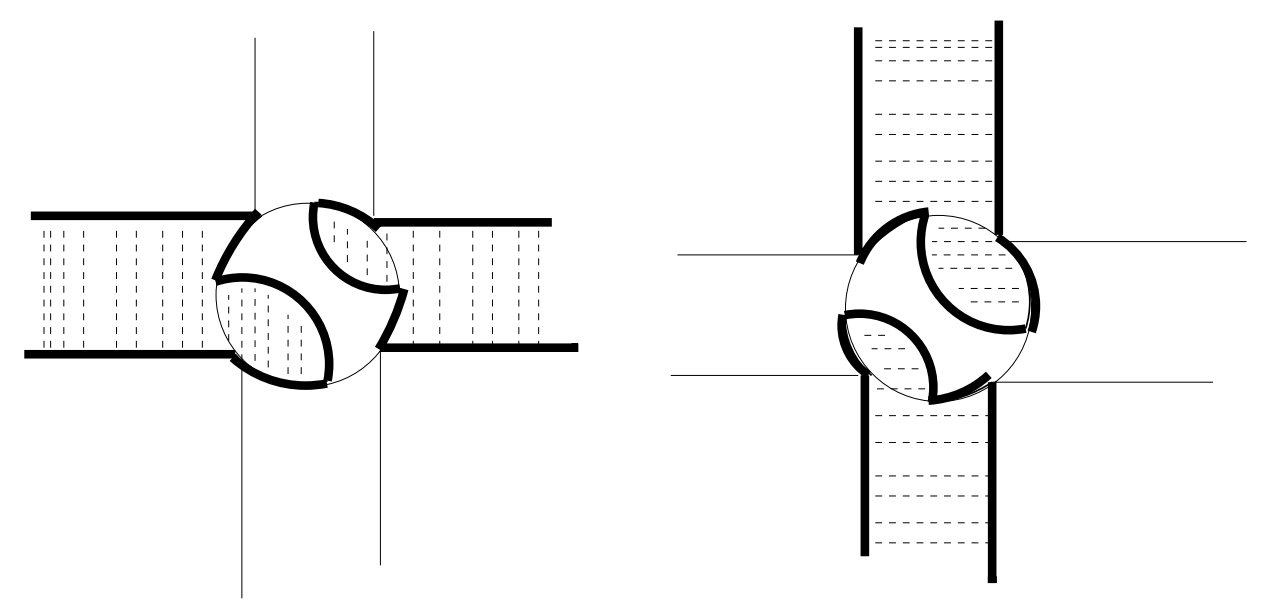

FiguRE 7. Wrong dividing set.

The proof of Proposition 4 is complete.

Returning to the Lefschetz fibration construction, we can now obtain a required decomposition of a Stein manifold $W$ without 1-handles: we represent $W$ as a Legendrian surgery cobordism for a Legendrian link $L$, use Proposition 4 to find an appropriate open book, and add to the fibration a Lefschetz handle corresponding to the positive Dehn twist along a component of $L$ for each Legendrian 2-handle of $W$ (see [AO1]); note that Lefschetz fibration given by the torus knot (in the absence of L) produces the (unique) Stein structure on $D^{4}$.

For the case where 1-handles are present, we combine the argument from [AO1] with Proposition 4. The Stein manifold is represented as a Legendrian surgery on a link in $\#_{n} S^{1} \times S^{2}$, which in turn corresponds to a diagram consisting of a Legendrian link in $S^{3}$ and $n$ dotted circles for the 1-handles. We first use Proposition 4 to find a "nice" open book for $S^{3}$, and then for each dotted circle we scoop a disk out of each page, so that the open book represents $\#_{n} S^{1} \times S^{2}$ now, and the Legendrian link is contained in a page. As before, we add Lefschetz handles compatibly with Legendrian handles. The pages of resulting open book will have multiple boundary components; we need an open book with a connected binding to use the Ozsváth-Szabó definition of contact invariants, so we make the boundary of the page connected by plumbing in some positive Hopf bands (for the Stein fillings, this corresponds to taking the boundary connected sum with the Stein ball $D^{4}$ ).

\section{Acknowledgements}

I am grateful to Peter Kronheimer for many helpful discussions, and to Paolo Lisca for pointing out a gap in the earlier version of this paper. 


\section{References}

[AO1] S. Akbulut and B. Ozbagci, Lefschetz fibrations on compact Stein surfaces, Geom. Topol. 5 (2001), 319-334, erratum Geom. Topol. 5 (2001), 939-945.

[AO2] S. Akbulut and B. Ozbagci, On the topology of compact Stein surfaces, Int. Math. Res. Not. (2002), 769-782, erratum Geom. Topol. 5 (2001), 939-945

[Bi] J. Birman, Braids, links and mapping class groups, Annals of Math. Studies 82, Princeton Univ. Press, Princeton, NJ, 1974.

[El] Ya. Eliashberg, Topological characterization of Stein manifolds of dimension $>2$, Internat. J. Math. 1 (1990), 29-46.

[Gi1] E. Giroux, in preparation.

[Gi2] E. Giroux, Convexité en topologie de contact, Comment. Math. Helv. 66 (1991), 637677.

[Go] R. Gompf,Handlebody construction of Stein surfaces, Ann. of Math. (2) 148 (1998), 619-693.

[GS] R. Gompf and A. Stipsicz, 4-manifolds and Kirby calculus, Graduate Studies in Mathematics, 20, AMS, 1999.

[Ho] K. Honda, On the classification of tight contact structures I, Geom. Topol. 4 (2000), 309-368.

[Ka] W. Kazez, A cut-and-paste approach to contact topology, ArXiv:math.GT/0210091.

[KM] P. Kronheimer and T. Mrowka, Monopoles and contact structures, Invent. Math. 130 (1997), 209-255.

[LM] P. Lisca and G. Matić, Tight contact structures and Seiberg-Witten invariants, Invent. Math. 129 (1997), 509-525.

[LS] P. Lisca and A. Stipsicz, in preparation.

[OS1] P. Oszváth and Z. Szabó, Holomorphic disks and topological invariants for closed 3manifolds, ArXiv:math.SG/0101206.

[OS2] P. Oszváth and Z. Szabó, Holomorphic disks and 3-manifold invariants: Properties and Applications, ArXiv:math.SG/0105202.

[OS3] P. Oszváth and Z. Szabó, Holomorphic triangles and invariants for smooth 4-manifolds, ArXiv:math.SG/0110169.

[OS4] P. Oszváth and Z. Szabó, Absolutely graded Floer homologies and intersection forms for four-manifolds with boundary, Adv. Math. 173 (2003), 179-261.

[OS5] P. Oszváth and Z. Szabó, Heegaard Floer homologies and contact structures, ArXiv:math.SG/0210127.

[OS6] P. Oszváth and Z. Szabó, Holomorphic triangle invariants and the topology of symplectic four-manifolds, Duke Math. J. 121 (2004), 1-34.

[TW] W. Thurston and H. Winkelnkemper, On the existence of contact forms, Proc. Amer. Math. Soc. 52 (1975), 345-347.

Department of Mathematics, Harvard University, Cambridge, MA 02138

E-mail address: olga@math.harvard.edu 